\title{
Medical Image Processing: Detection and Prediction of PCOS - A Systematic Literature Review
}

\author{
Siji Jose Pulluparambil ${ }^{1}$ \& Subrahmanya Bhat ${ }^{2}$ \\ ${ }^{1}$ Research Scholar, College of Computer Science and Information Science, Srinivas University, \\ Mangalore, India. \\ Orcid ID: 0000-0001-6214-3304; E-mail: sijijohn2223@gmail.com \\ ${ }^{2}$ Professor, College of Computer Science and Information Science, Srinivas University, Mangalore, India. \\ Orcid ID: 0000-0003-2925-1834; E-mail: itsbhat@ gmail.com
}

Area/Section: IT in Health Sciences.

Type of the Paper: Literature Review.

Type of Review: Peer Reviewed as per $|\mathrm{C}| \mathrm{O}|\mathrm{P}| \mathrm{E} \mid$ guidance.

Indexed in: OpenAIRE.

DOI: $\underline{\text { https://doi.org/10.5281/zenodo. } 5782763}$

Google Scholar Citation: IJHSP

\section{How to Cite this Paper:}

Pulluparambil, Siji Jose, \& Bhat, Subrahmanya, (2021). Medical Image Processing: Detection and Prediction of PCOS -A Systematic Literature Review. International Journal of Health Sciences and Pharmacy (IJHSP), 5(2), 80-98. DOI: https://doi.org/10.5281/zenodo.5782763

International Journal of Health Sciences and Pharmacy (IJHSP)

A Refereed International Journal of Srinivas University, India.

Crossref DOI : $\underline{\text { https://doi.org/10.47992/IJHSP.2581.6411.0075 }}$

(C) With Author.

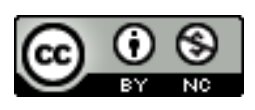

This work is licensed under a Creative Commons Attribution-Non-Commercial 4.0 International License subject to proper citation to the publication source of the work.

Disclaimer: The scholarly papers as reviewed and published by the Srinivas Publications (S.P.), India are the views and opinions of their respective authors and are not the views or opinions of the SP. The SP disclaims of any harm or loss caused due to the published content to any party. 


\title{
Medical Image Processing: Detection and Prediction of PCOS -A Systematic Literature Review
}

\author{
Siji Jose Pulluparambil ${ }^{1}$ \& Subrahmanya Bhat ${ }^{2}$ \\ ${ }^{1}$ Research Scholar, College of Computer Science and Information Science, Srinivas University, \\ Mangalore, India. \\ Orcid ID: 0000-0001-6214-3304; E-mail: sijijohn2223@gmail.com \\ ${ }^{2}$ Professor, College of Computer Science and Information Science, Srinivas University, Mangalore, India. \\ Orcid ID: 0000-0003-2925-1834; E-mail: itsbhat@gmail.com
}

\begin{abstract}
Purpose: Considered as the most common hormonal disorder among women, polycystic ovary syndrome or PCOS affects 1 in 10 reproductive aged women (18 - 44 years). Ultrasonography is applied for assessing the ovaries to detect PCOS. The patients affected by PCOS consist of 10-12 cysts present in the ovary, but more than 10 cysts are more enough to diagnose the disorder from the ultrasound images. Then, by examining the ultrasound the presence of follicles will be determined. Therefore, the image processing approaches have assisted to identify the characteristics like follicle size, number of follicles and structure to minimize the workload and time of doctors. PCOS do not have better treatment and effective diagnosis. This paper includes reviewing a summary of some of the researches that have been going in area of medical diagnosis. Based on the review, research gap, research agendas to carry out further research are identified.
\end{abstract}

Approach: A detailed study on the algorithms used in medical image processing and classification. Findings: The study indicated that most of the classification of polycystic ovarian syndrome is done merely on the clinical data sets. The new hybrid methodology proposed will be more precise as both images and lifestyle are analysed.

Originality: The type of data required for detection system are studied and the architecture and schematic diagram of a proposed system are included.

Paper Type: Literature Review.

Keywords: PCOS, Polycystic Ovary Syndrome, Contrast Enhancement, ABCD Analysis, CAD, SVM, PCA.

\section{INTRODUCTION :}

Polycystic ovary syndrome [1-3], commonly known as PCOS, is regarded the most common hormonal disorder among women which affects 1 in 10 women of childbearing age from 18 to 44 years. This disorder was first identified by Leventhal and Stein in 1935. PCOS is an endocrine disorder [4] with symptoms such as enlarged ovaries and, in some cases, cysts along the outer edges of ovary. It is considered the leading cause of female infertility. Both luteinizing hormone and follicle stimulating hormone are essential for the maturation of egg in the ovaries [5, 6] and PCOS condition disrupts the balance of these hormones in the body. Apart from enlargement of ovaries, another distinct characteristic of PCOS disorder in female reproductive system is the development of follicular cysts and the formation of pre-mature follicles in the ovary. The women affected by PCOS can undergo main symptoms such as prolonged or irregular menstrual periods, polycystic ovarian structure and contains excess androgen (male hormone) level and also includes some other symptoms such as obese, unwanted growth of hairs on face and body, hyperinsulinemia, unusual weight gain etc. Moreover, the exact reason for PCOS is unknown [7], but excess insulin, heredity, low-grade inflammation and excess androgen are some of the factors that cause PCOS.

In PCOS [8], early and accurate diagnosis is more significant because it has the ability to cause other diseases namely uterine cancer, diabetes, heart disease, insulin resistance etc. Therefore, to avoid other such diseases diagnosis and treatment at the early stage should be initiated. Medical imaging has an important role to diagnose the disorder. PCOS can be of four types - insulin resistant PCOS, pill-induced PCOS (post-pill PCOS), inflammatory PCOS and hidden PCOS. In recent days, several medical imaging methods namely, Magnetic Resonance Imaging (MRI), Computerized Tomography (CT), X-Ray and Ultrasonography are 
introduced to form pictures of human body for detecting the symptoms, providing treatment and monitoring the medication progress $[9,10]$. Among the imaging methods, ultrasonography is predominantly applied for imaging uterus, kidney and liver since it is non-invasive and less radiated imaging method and also it is adaptable, transferable, portable, inexpensive and has better temporal resolution. Therefore, ultrasonography is applied for assessing the ovaries to detect PCOS [11].

Ultrasonography uses ultrasound (high-frequency sounds waves) of 2-15 MHz frequency for generating the images of tissues and other internal organs [12]. The ultrasonography is considered as inexpensive, very safe and painless, and it can be utilized even pregnancy. In ultrasound device, two-dimensional ovary images in grey and white/black colour are generated. The generated polycystic ovarian and normal ultrasound images have been different from one another $[13,14]$. Normally, the patients affected by PCOS consist of 10-12 cysts present in the ovary, but more than 10 cysts are more enough to diagnose the disorder from the ultrasound images. Then, by examining the ultrasound the presence of follicles will be determined. Further, detecting the follicles by physicians through manual examination takes more time and formidable [15-17] because the ultrasound image contains speckle noise and artefacts. This is due to the presence of blood vessels, endometrium and tissues in the ovary that are also captured during the ultrasound scanning process. Therefore, the image processing approaches have assisted to identify the characteristics like follicle size, number of follicles and structure to minimize the workload and time of doctors [18].

Several artificial intelligence-based approaches utilizing support vector machine (SVM), artificial neural network (ANN) and fuzzy logic have been proposed to classify follicles and diagnose PCOS [19-21]. Machine learning is the application of artificial intelligence in uses cases such as data prediction, image recognition and detection etc. It also provides the system, the ability to learn and enhance itself from experience. Logistic regression (LR), K-nearest neighbours, linear discriminant analysis (LDA), classification and regression trees (CART), random forest classifier and naive Bayes classifier are some of the machine learning algorithms used in segregating non-PCOS and PCOS samples [22-24]. Gabor wavelet model is the most used approach for feature extraction. However, these existing methods have less follicle detection rate, high misidentification rate and consumes more time to filter the image [25]. Therefore, it is very important to develop new methods for detecting the ovarian dysfunction in the initial stage to avoid the consequences since, it is the major reason for infertility and no cure has been identified yet [26]. Deep learning is one of the progressing technologies that can solve many problems in different fields. It supports researchers and healthcare professionals to detect the hidden data. Besides, it supports doctors for analysing the types of disorder accurately and then assist to provide treatment better. However, PCOS do not have better treatment and effective diagnosis, so an effective deep learning method is employed for detecting the PCOS patients with higher accuracy rate [27-30].

\section{RESEARCH OBJECTIVES :}

Our research area is on medical image processing using deep learning techniques. Automatic diagnosis with high accuracy is our goal. Based on that intention we developed a few target questions.

(1) How the medical sector will be benefited with the development of new classification methods?

(2) How different image and data analytics solutions prove themselves to be best?

(3) How to develop the most accurate, reliable, efficient, flexible and cost-effective systems that contributes to the requirement?

(4) What implications these findings have?

(5) What kinds of data are needed to achieve an automated diagnosis system?

(6) How to design proposed architecture?

(7) To find out the research gap and research agendas to contribute further research.

\section{METHODOLOGY USED :}

The proposed study uses the method of collecting the data to review the literature on the topic. The data required for the study was collected from secondary data, research papers, books, journals, and magazines which are available online. A Comparative study was done and the issues in the existing systems were identified.

\section{RELATED WORK ON PCOS DETECTION :}

Different existing methods used for detecting PCOS have been discussed in this section. PCOS is one among the most commonly occurring endocrine disorders affecting women of reproductive age. It can cause many 
problems and hinders the fertile nature of females; therefore, to live a good life, females must be aware of PCOS. Nearly, $70 \%$ of the PCOS patients are unidentified. In earlier days, adaptive K-means clustering methods had been introduced to segment the follicles using ultrasound images. However, this clustering method did not focus on normal and abnormal classification. Therefore, N.S. Nilofer \& R. Ramkumar [31] proposed the use of an artificial neural network (ANN) model, combined with improved fruit fly optimization approach (IFFOA). The IFFOA-ANN model resizes the ultrasonic input images and eliminates the noises for improving the quality of input image. Further, adaptive k-means clustering method was employed to segment the follicles. Then, to employ a feature extraction technique, statistical GLCM has utilized. Finally, ANN would be trained for better classification. As a result, better precision, recall and accuracy had achieved.

In [32], the authors C. Gopalakrishnan and M. Iyapparaja implemented an active contour with modified Otsu technique to extract the follicle for automatically detecting PCOS from the ultrasound image. The modified Otsu technique had applied to binarize the ovary image for segmentation process. The binary mask comprised of foreground and background regions neat to segmented object, therefore the contour initialization issues were solved. Further, thresholding scheme had infused to extract the objects from the ovary image depending on the distributed gray level. Finally, better result had attained in terms of accuracy. Therefore, the modified Otsu technique was suitable for follicle extraction.

Recently, image recognition methods could be employed in medical fields to diagnose and classify the diseases. However, PCOS detection based on traditional approaches had some disadvantages so, Dongyun He et al. [33] introduced a probabilistic approach to diagnose the disease by employing learned quality kernel. To attain proper cues discovery, the training images were segmented initially into different equal-size grids. Further, based on the texture features and greyscale, every grid on the input image was approximately resembled by a quality score. Subsequently, every image could be described as a score matrix. By considering the score matrix, the statistical based model could be leveraged providing the feature vectors. Finally, the determined feature vectors were learned by employing the probabilistic model and then the learned feature vectors had been transformed into SVM kernel to detect PCOS. As a result, the probabilistic approach had enhanced the detection of PCOS.

C. Gopalakrishnan \& M. Iyapparaja [34] presented scale-invariant feature transform (SIFT) descriptors model to detect the PCOS from the ultrasound images of ovary. Here, canny edge detection scheme had used initially to improve the image quality and detect follicles edge from the ultrasound image. Pre-processing, gradient calculation, non-maximum suppression and thresholding were the process performed in canny edge detection scheme. Then, SIFT descriptors could be employed to determine the feature descriptors for recognizing the syndrome. Eventually, data training and classification were carried out by deploying support vector machine (SVM). As a result, better accuracy, mean squared error and normalized absolute error had attained for detecting the PCOS.

In women reproductive system, infertility is one of the major problems that is caused by the inhibition of the maturation process of follicles, thereby affecting the count of follicles present in the ovary. Knowing the size and number of follicles can be helpful in the diagnosis of PCOS. Therefore, the detection process requires maximum accuracy and it takes more time. Normally, feature extraction and classification or stereology calculation is employed to detect PCO. R M Dewi et al. [35] introduced a competitive neural network model to detect polycystic ovary from ultrasound images given as input. For feature extraction, Gabor wavelet model was used and the competitive neural network acted as the classifier. The competitive neural network proposed in the paper used a combination of Max net and Hemming net so, the data classification could be carried out using specific features of ultrasound data. Finally, better accuracy and less time had attained.

Basically, PCOS is a major reproductive issue, which leads to irregular menstruation periods for women due to the changes in androgen hormone and insulin. It must be detected at the early stage since it has the capability to cause other diseases namely, obesity, diabetes and cardio vascular problems. PCOS was mainly detected by hormone test or analysing ovary morphology, but the detection using ultrasonography would be a more suitable way. Therefore, I F Thufailah et al. [36] introduced Elman neural network using ultrasound images to classify PCOS. Gabor wavelet was chosen as the feature extraction model, and then for non-PCOS and PCOS classification, Elman neural network was employed. The previous conditions could be easily recalled due to the context layer on the Elman neural network. As a result, better accuracy had achieved using 32 features.

PCOS has been considered as one of the prominent disorders that leads to infertility around the world, however it could be prevented if the disease was detected at the earlier stage. To diagnose PCOS, the recognition of follicles from the ultrasonic image of ovary was essential. But the manual methods were 
inconvenient, time consuming, error-prone and laborious to detect the follicles. Also, many existing methods were limited by less sensitivity, accuracy and specificity because of jagged and irregular follicle edges. Therefore, O.R. Isah et al. [37] proposed an efficient hybrid model using particle swarm optimization (PSO) along with an ANN for the automatic classification of follicles. The input ultrasonic images, initially, undergo de-speckling using lee filter. Then, many features were extracted from the ultrasonic image. Moreover, PSO algorithm had been employed to close twelve of the most significant. Finally, the chosen features had been fed into the multilayer perceptron ANN for training and testing propose. As a result, better sensitivity, accuracy and specificity were achieved.

Untari N. Wisesty et al. [38] proposed a modified back propagation algorithm to detect the PCOS using the ultrasound images. Generally, the follicle detection of PCO was mainly based on stereology calculation or feature extraction and classification. Here, the detection of PCOS was based on feature extraction and classification. The modified back propagation model was used as a classifier whereas the Gabor wavelet had considered for feature extractor. Conjugate Gradient- Fletcher Reeves and Levenberg- Marquardt optimization was the modified back propagation algorithm. However, the maximum accuracy had attained using Levenberg- Marquardt optimization by considering 16 vector feature and 33 neurons.

E. Setiawati et al. [39] presented a PSO model to segment the follicles for assisting PCOS detection. Here, new clustering model had developed using PSO along with modified non-parametric fitness function to segment the follicles. Error detection based on pixel value and increasing the similarity on human vision would be the main objective of the fitness function. Then, to generate convergent and compact clusters, normalized mean square error (NMSE) and mean structural similarity index (MSSIM) techniques were utilized by the modified non-parametric fitness function. Finally, more convergent solutions were produced by the PSO fitness function. Further, the extracted follicular size and contrast enhancement had also influenced by the PSO performance also investigated.

H. Prasanna Kumar \& S. Srinivasam [40] introduced Chan-vase model along with split-bregman optimization using ultrasound images for fast segmentation of PCOS. The diagnosis of PCOS with ultrasound images would provide significant information about the size and number of follicles. The small follicles could be determined using enhanced active contour without edge model. Moreover, the accuracy of the segmented images and computation time were improved by employing split-bregman optimization model. The outcomes showed that the split-bregman optimization model has obtained better result with reduced computation time and iteration.

\subsection{Phases in Image Processing:}

Feature selection and extraction are important processes in all CAD (Computer Aided Detection) systems. Features can be of four - texture, morphologic, model based and descriptor features [46].

\section{(1) Texture:}

In the ultrasound CAD framework, the texture element is the most commonly used feature. Analysing the texture elements can reveal the characteristics of the affected area. A partial list of commonly used features is produced below.

(1) Laws' Texture Energy (LTE) - LTE makes use of local masks to identify the type of texture [47]. The mask's window size is generally set to $5 \mathrm{x} 5$. The local masks are applied to the image to compute texture energy which are further represented in vector form.

(2) Contrast of Gray Level Values - The local variations in the input image can be identified using this feature.

(3) Gray Level Cooccurrence Matrix (GLCM) - GLCM feature can be used to study the frequency of cooccurring pixels with specified grayscale values. CAD systems make extensive use of GLCM as a feature $[47,48]$.

(4) Wavelet Features - Applying wavelet transforms to the ultrasound image can create new features for image detection. Wavelet transform has become a popular technique in image processing, including ultrasound image processing.

(2) Morphology:

The morphologic feature differs from the texture features on the grounds that the former tends to be more focused on the lesion or the visible features of the affected area.

(3) Descriptor Features:

The features that are described or set by clinical experts are known as descriptor features. There cannot be a standard set of descriptor features, as they are specific to the application. 
Shape, calcifications, posterior echo, posterior shadow and echo characteristics are the common descriptor features used, while considering ultra sound images for growth detection. To identify cysts in ovary also similar descriptor features are analysed. In this study shape does not have a role. Calcifications help to know about the presence or absence of the symptoms [47].

\section{(4) Classifiers:}

After appropriate features are identified and extracted, a classifier has to be built segregate the images.

(1) Linear classifier - A linear classifier refers to the approach in machine learning which makes use of a linear collection of feature values to perform classification. The dataset in this case includes features of each class being considered in the problem. From the training data, a hyperplane can be formed, where one region of the plane predicts one class and another region predicts another class. The features of a class are merged to form the equation of a line that marks the decision boundary for that particular class. These classifiers are suitable when the data is linearly separable.

Linear classifiers are best suited in problems similar to the Iris classification data represented in figure 1, which considers two features sepal width and sepal length.

For $n, c \in N$, a set of $c$ labels $I$, and a sequence $x^{i} \in \mathbb{R}^{n}, y^{i} \in I, 1<i<M$, one calls the problem of finding a function $f: \mathbb{R}^{n} \rightarrow I$ such that $f\left(x^{i}\right)=y^{i}$ for all $1 \leq i \leq N$ an $n$-dimensional classification problem withcclasses.

- $\quad$ The $\operatorname{set}\left(x^{i}, y^{i}\right)_{1 \leq i \leq M}$, is called training data or pre labelled data.

- If $c=2$, one referes to it as a binary classification problem.

- The problem is called a linear classification problem, if the given data points $x^{i}$ can be separated according to their respective labels $y^{i}$ by means of hyperplanes. If such a separation is impossible, the problem is considered non-linear.

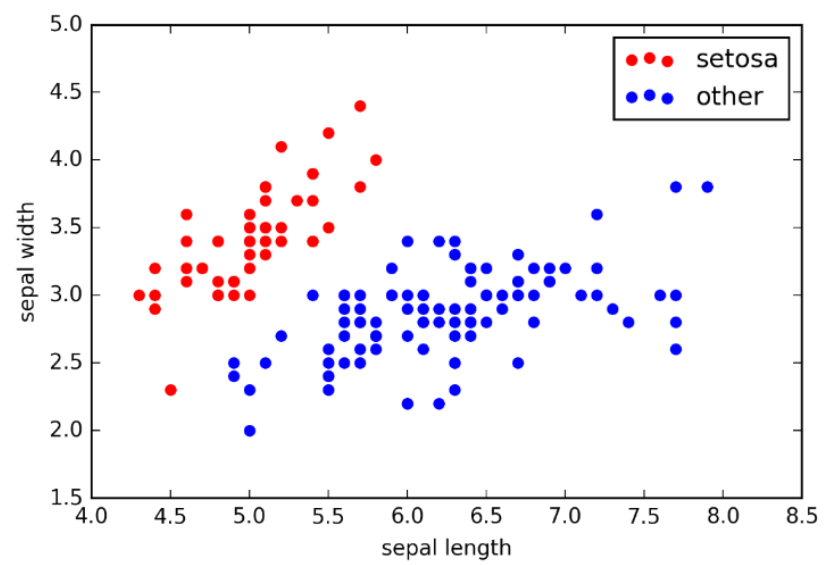

Fig. 1: Iris flower dataset

Some of the algorithms used in linear classification are logistic regression, Support Vector Machine (SVM) and perceptron models.

(2) Bayesian classifier - Generally speaking, a Bayes classifier or Bayesian classifier is a probabilistic classifier that applies the Baye's theorem. Such a classifier can be constructed by computing the conditional probability of events or in this case, the probability of the given feature vector $X$ to belong in class $y$.

A Bayesian classifier can predict feature values of the member of the class, if the class is known beforehand. If class is unknown, it can be predicted using the known feature values. From the training data, a probabilistic model of the features is built, which can be used for classification.

The simplest version of Bayesian classifier is a naive Bayes classifier. It is considered naive because it makes overly simplistic assumptions which may not be correct in real-world data. The assumptions are-

- There is zero correlation between the features, which means the value of one feature is not dependent on the value of any of the other features.

- $\quad$ All features are given equal weightage or importance.

Naive Bayes classifiers can be categorized into Gaussian naive Bayes classifier, Multinomial naive Bayes classifier and Bernoulli naive Bayes classifier.

(3) Support Vector Machine - Another example of linear classifiers, the support vector machine or SVM is a simple, computationally low demanding, supervised learning algorithm which can handle regression as well 
as classification. Each data item gets plotted as a point in an n-dimensional space and the optimal hyper plane that can linearly separate the classes has to be identified. Here, $\mathrm{n}$ is the number of features being considered in the problem. When data points in the training data are plotted on to an n-dimensional space as shown in figure 2 , there is the possibility of multiple hyper planes that can separate the different classes.

The objective is to identify a plane that maximises the margin. Margin refers to the distance between data points of the different classes.
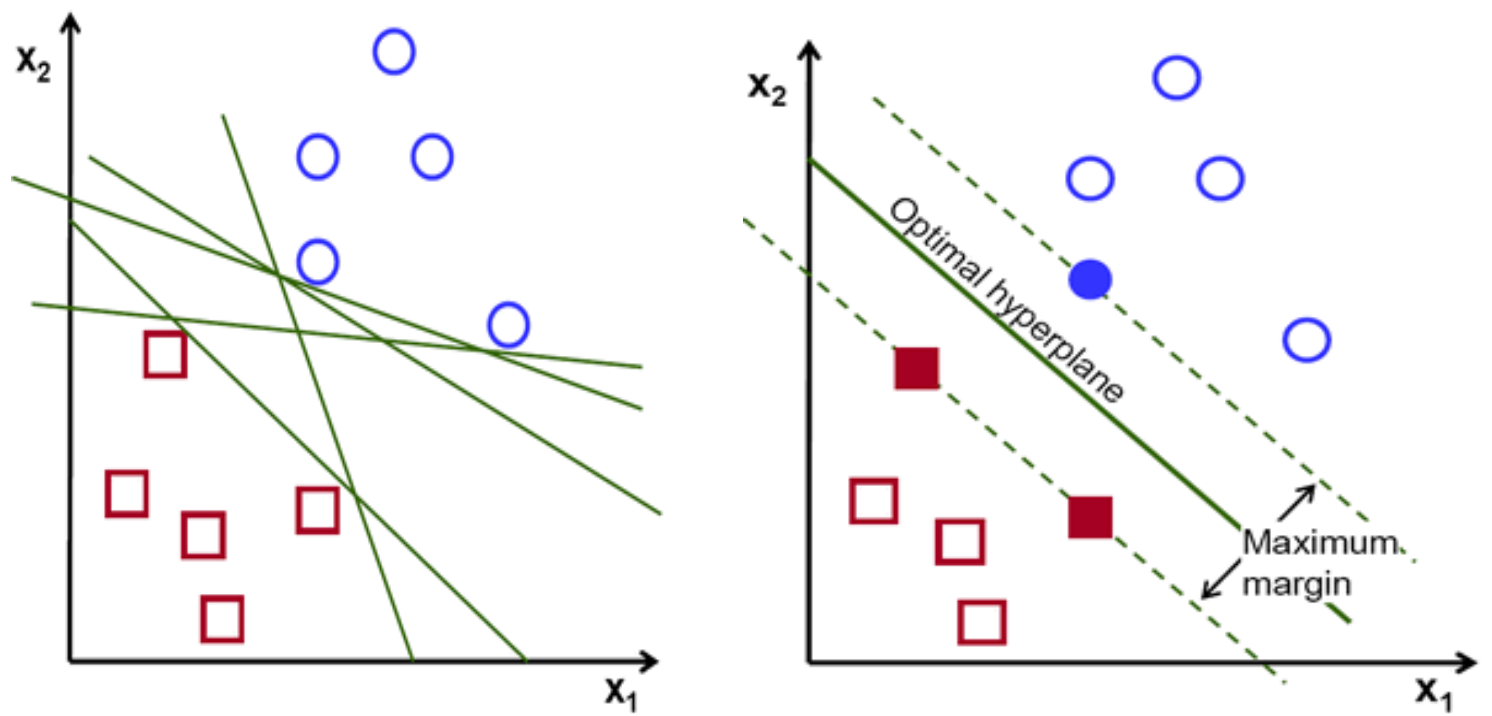

Fig. 2: Plotting on Hyper planes

(4) Decision tree - Decision tree is a non-parametric, supervised ML algorithm, and can be applied to both regression and classification problems. As the name implies, the classifier is constructed using a tree like structure, where a node represents a condition involving one or more features and the edges represent the outcomes of the condition and the decision to be taken in each case. The algorithm is recursive, meaning that a node can have child nodes, representing sub conditions. The topmost node in a decision tree, which divides the whole data into two or more sets based on the value of features, is considered the root node. When visualized, decision trees look similar to a flowchart and decision regarding the given input data can be taken by navigating through the tree from the root to a terminal node according to the input data and decision nodes of the tree.

The algorithm for constructing a decision tree can be summarized as -

(1) Initially, the whole of training dataset is positioned as the root, which will later be partitioned into child nodes.

(2) Feature values in the dataset have to be categorical. In case of continuous values, they can be converted to categorical values.

(3) Each record in the dataset has to be inserted into the tree, in a recursive manner, until one of the conditions is met

(4) All the tuples belong to the same attribute value.

(5) There are no more remaining attributes.

(6) There are no more instances.

Decision tree classifiers are simple to understand and interpret. With the visualized model of a decision tree, it is easy to understand the logic behind a decision. Decision tree algorithm is capable of working with unclean data or data with missing values. The selection of the feature/attribute to be placed at the root or as nodes at the subsequent levels is not done randomly. The 'attribute selection problem' is solved by computing values of criteria such as Gini index, information gain, gain ratio, entropy, Chi-Square, and reduction in variance. Typically, the attribute with a high value is placed at the root. The use of Gini index for attribute selection can be understood from the decision tree visualized in figure 3 .

A decision tree's accuracy is greatly influenced by the process of splitting data into nodes and sub-nodes. There are multiple algorithms which can be used to construct decision trees, based on the type of attribute selection criteria used and the type of target variable (discrete or continuous). Some of the algorithms used in 
decision trees are ID3 (Iterative Dichotomiser 3), CART (Classification and Regression Tree), C4.5, MARS (Multivariate Adaptive Regression Splines) and CHAID (Chi-square Automatic Interaction Detection).

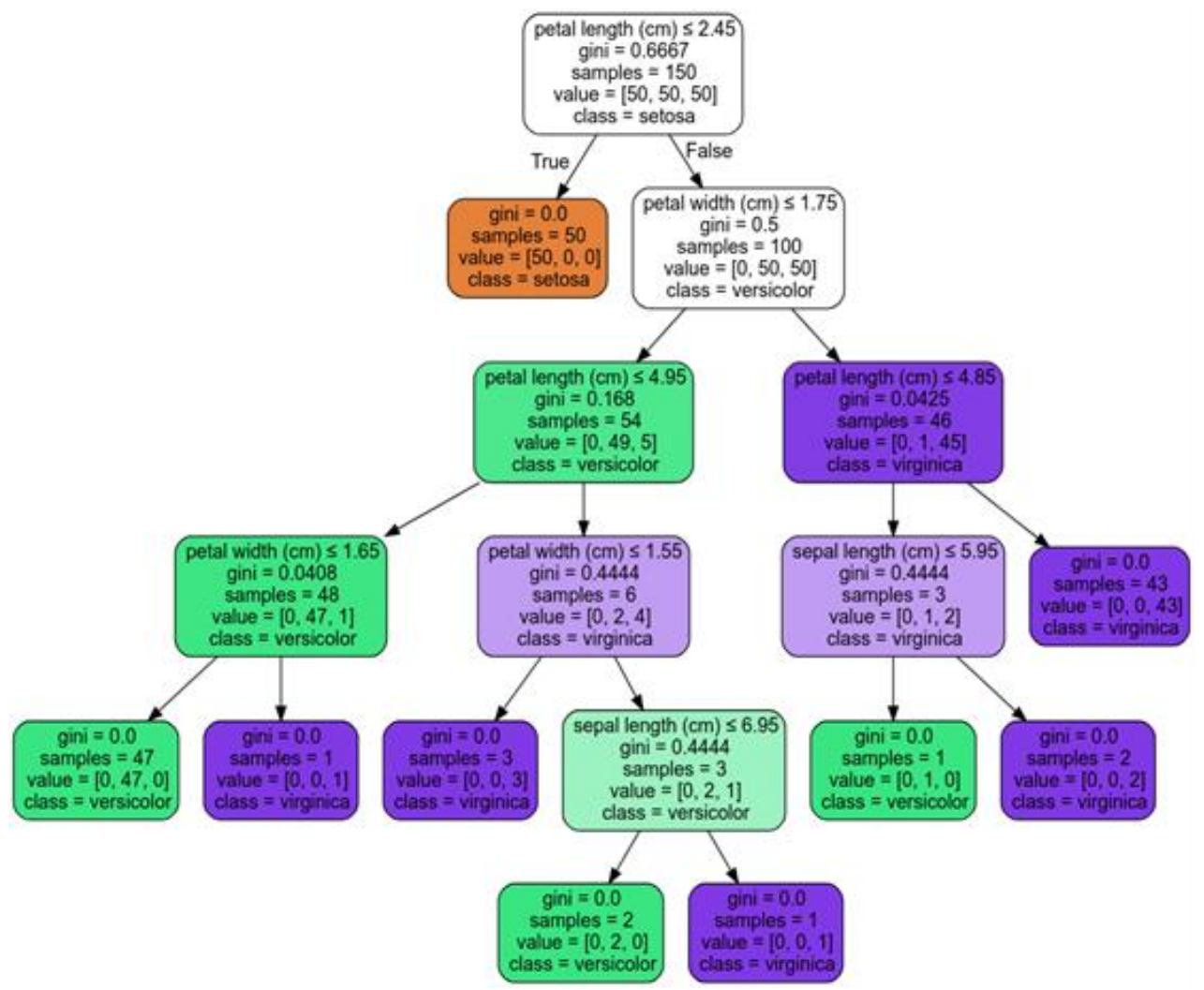

Fig. 3: Decision Tree Structure

(5) Artificial Neural Network - Artificial neural network (ANN) refers to that class of algorithms and models that are based on artificial neurons, which works in the same manner as the neurons in the human brain. Artificial neural networks or simply neural networks work similar to the human brain and can recognize patterns and relationships from the data fed into it.

A neural network, in its most basic form, requires at least three layers - input layer, hidden layer and output layer. Information given by the user is captured by the input layer or input nodes. The information so received at the input layer is forwarded to the succeeding layer, the hidden layer. Hidden layers are where computations are performed on the inputs to extract features and identify patterns. The number of hidden layers present in a neural network determines its complexity. The output layer communicates the output, usually the classification result. There can be multiple nodes in the output layer. For a binary classification problem, there needs to be two nodes in the output layer. A problem with 10 classes requires 10 nodes and so on. Therefore, it can be stated that the number of nodes in the output layer equals the number of classes considered for the problem.

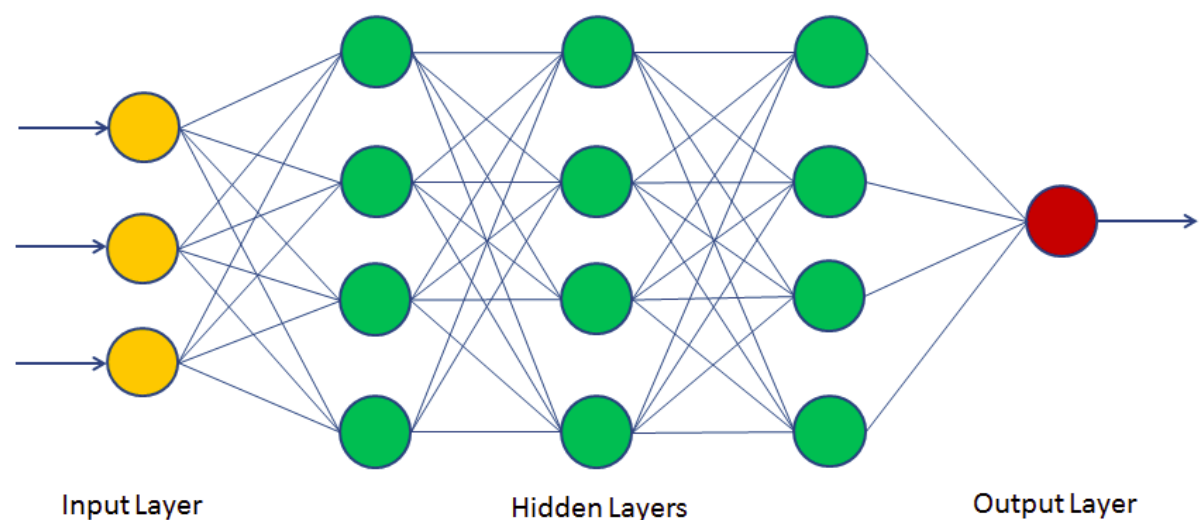

Fig. 4: ANN Structure 
The above diagram represents a neural network model with 3 input nodes, 3 hidden layers with 4 nodes each, followed by an output layer with 1 output node.

An artificial neural network performs weighted summation of the given inputs and adds a bias value. This computation can be represented as

$$
\sum_{n}^{i=1} w_{i} * x_{i}+b
$$

where, $\mathrm{x}$ refers to input and $\mathrm{w}$ refers to its corresponding weight. Each neuron has an activation function defined for it, which determines whether a neuron should be activated, based on the relevance of the input processed by the neuron in the decision making process. Activation functions provide non-linearity to neural networks. Commonly used activation functions are identity function, sigmoid function, softmax, ramp function and Rectified Linear Unit Function (ReLU). There are different variations of neural networks such as Feed Forward (FF) networks, Convolutional Neural Network (CNN), Recurrent Neural Network (RNN), Long Short-Term Memory (LSTM) Network, Radial Basis Function (RBF) Neural Network, Auto Encoder (AE), Extreme Learning Machine (ELM), Hopfield Network (HN), Generative Adversarial Network (GAN), etc.

\section{ALGORITHMS ANALYSED FOR STUDY :}

The process of image processing requires high quality images with high resolution. A few algorithms which can be used for this purpose are discussed below.

\subsection{Contrast Enhancement Algorithms:}

Noise elements in images (edges, contrast boundaries, etc.) reduces the overall quality of image. To eliminate these undesired elements and sharpen the image, contrast enhancement (CE) is applied. Even though contrast enhancement is achieved through digital manipulation of images, it improves the visual quality, as perceivable by our eyes. Over the years, several contrast enhancement techniques have been proposed which have applications in different domains of study. Commercially used contrast enhancement algorithms are -

(1) Histogram equalization (HE):

This is a non-linear contrast enhancement algorithm, wherein the mean values of spectral bands are calculated and the pixels are modified so as to flatten or equalise the histogram. Histogram equalization is based on the idea that for an image with the best visual appearance, its histogram should look like the regular distribution. For this purpose, Cumulative Distribution Function (CDF) is computed. CDF is the fraction of pixels which has intensity value less that equal to a specified limit. The HE process is straight forward and the results can be easily interpreted. Histogram equalization works better on grayscale images and therefore ideally suited for pre-processing $\mathrm{x}$-rays and medical images.

\section{(2) Adaptive Histogram Equalization:}

This is an improvement of the HE algorithm, where instead of a single histogram for the whole image, several histograms are computed for separate parts of an image and the intensity values are adjusted based on them. An image is divided into several sections known as tiles and a histogram is computed for each such tile. The contrast of a tile can be enhanced by pixel redistribution, based on the histogram of the tile. To soften the boundaries between adjacent tiles, bilinear interpolation is used. Contrast Limited Adaptive Histogram Equalization (CLAHE) is an enhancement over traditional adaptive histogram equalization, wherein the computation of cumulative distribution function is preceded by partial clipping of the histogram.

\section{(3) Contrast stretching:}

In contrast stretching, the range of contrast values of the image is modified or stretched to fit the desired range of values. It is also called normalization, as we can lighten or darken images to match our requirements.

\subsection{Swarm Optimization K-means:}

Swarm optimization or particle swarm optimization (PSO) [49] is one of the bio-inspired algorithms and it is a simple heuristic to search and locate the optimal solution in the given solution space. PSO only requires an objective function and does not demand a gradient or any differential form of the objective. The PSO algorithm was proposed by Kennedy $\mathrm{J}$ and Eberrhart R C, inspired by the concept of swarm intelligence as seen in flocks of birds or schools of fishes.

Consider the analogy of birds searching for food. The birds fly in random directions in search for food and communicate or cooperate with each other to help their search. In PSO algorithm, a particle represents a bird. 
Each particle has characteristics such as velocity and fitness value. The particles search in the D dimensional problem space by communicating their findings to the nearby particles, thereby converging to a near-optimal solution.

$\mathrm{K}$-means is an unsupervised learning algorithm used for clustering problems. The objective is to classify the given dataset into a set of clusters $\mathrm{C}=\left\{\mathrm{C}_{1}, \mathrm{C}_{2}, \ldots, \mathrm{C}_{\mathrm{k}}\right\}$. This classification into clusters is based on a set of $\mathrm{k}$ centroids computed on the basis of the dataset. Each point in the dataset is then assigned to the nearest centroid. When all the points are assigned, the first grouping is done. Compute the variance and place a new centroid for each cluster. The process of assigning points to the newly calculated centroids is repeated, until there are no changes in the assignment.

When particle swarm optimization algorithm is used in the K-means clustering, it is called Particle Swarm Optimization - K-means (PSO-KM).

The PSO-KM algorithm as presented in the 2005 paper [49] is described below.

(1) Initially, the data points are divided into k clusters. The position code of the particles is determined based on the centroid. Fitness value is computed and velocity initialized. To produce $\mathrm{N}$ particles, the process has to be repeated $\mathrm{N}$ times.

(2) Fitness value $\mathrm{f}\left(\mathrm{S}_{\mathrm{j}}\right)$ is computed with the position code of the particle. If the position is found to be better, the personal best position (Pbest $\mathrm{i}_{\mathrm{i}}$ gets updated and if required, the global best position (Gbest) as well.

(3) Recalculate the velocity and position of particles with (1) and (2)

(4) Apply K-means optimization to the new set of values. Re-cluster the data, find cluster centroids and compute fitness value and new position.

(5) When the position is optimal or the iterations have exceeded the maximum, the algorithm is terminated. Else, go back to step 2.

K-means optimization, with its better local search ability, allows faster convergence of PSO and improves the overall speed. There can be several variations of the algorithm applied to different scenarios like document classification, medical image classification, sentiment analysis, etc. There could be changes in the optimization techniques and distance calculation methods.

\subsection{Hybrid Principal Component Analysis:}

Principal component analysis (PCA) in image processing acts as an efficient tool for data analysis, which is found to be useful in several applications such as dimensionality reduction and data compression. When there is a large number of observations, PCA can be applied to synthesize a smaller set of variables (principal components), which captures most of the variances found in the original observations. PCA aims at reducing the number of components and produce a condensed or compact form of the data, without losing its general characteristics. The first principal component can provide the best representation of the data and its variances. Hybrid Principal Component Analysis could mean several things. In some contexts, it could mean the usage of PCA in association with a modified pre-processing, classification or clustering algorithms. It could also mean application of PCA in a new domain or use case. In some other context, it could mean the variations of the classical PCA. There are different flavours of PCA such as classical PCA, region based PCA, sub-pattern based PCA(SpPCA), cross-sub-pattern correlation based PCA (SubXPCA) and modular PCA. When an improvement is introduced in any of these existing methods or a combination of two or more methods is implemented, it can be considered hybrid PCA.

One such implementation can be found in a 2017 paper [50] for facial age estimation. They introduced two improved versions of existing PCA methods - extended SpPCA (ESpPCA) and extended SubXPCA (EsubXPCA). The ESpPCA method is baased on SpPCA, which uses a combination of local SpPCA feature and global PCA feature to arrive at the hybrid appearance feature. The ESubXPCA is an improvement over SubXPCA, which focuses on hybrid appearance variations. ESpPCA feature set is evaluated first and applies classical PCA on it. The diagram below compares the different PCA methods side by side. 


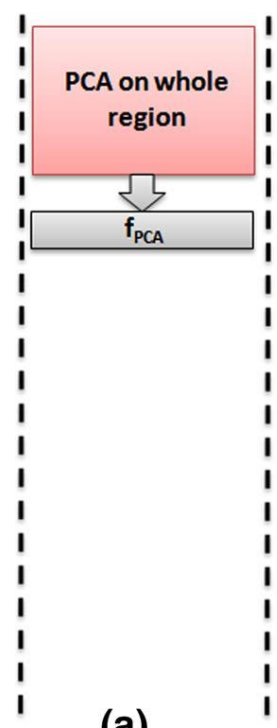

(a)

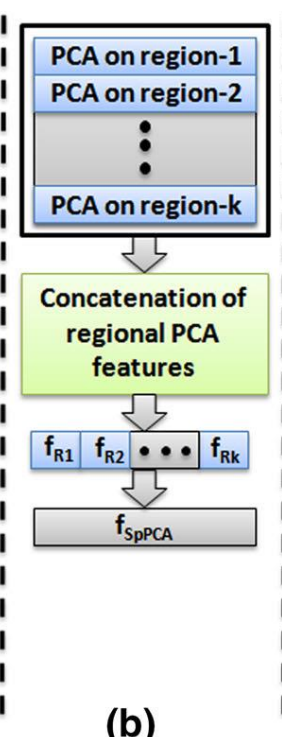

(b)

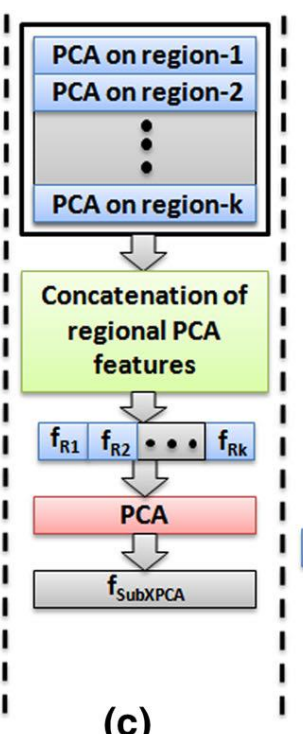

(c)

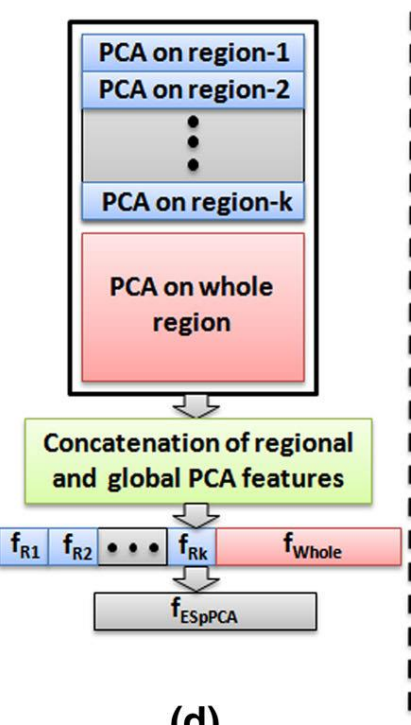

(d)

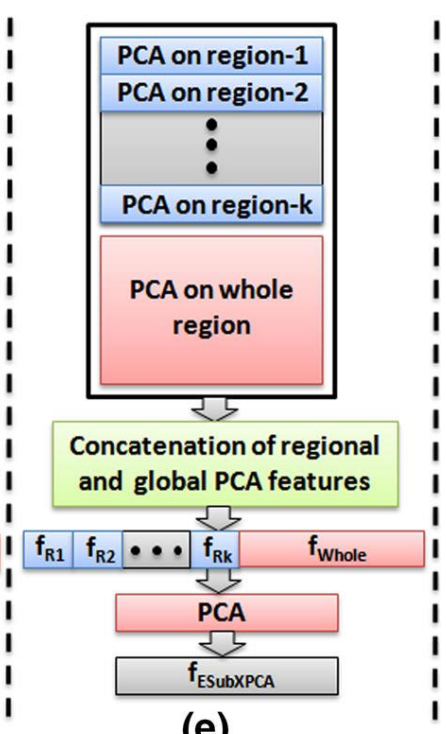

(e)

(a) Classical PCA (b) SpPCA (c) SubXPCA (d) ESpPCA (e) EsubXPCA

Fig. 5: Different PCA methods

\section{ANALYSIS ON MEDICAL IMAGE PROCESSING TECHNIQUES :}

Medical Image Processing as well as classification algorithms is an integral component of modern diagnosis systems. The success and failure of systems are largely dependent on implementing the best method opted for the image processing and classification. Table 1 below depicts the name of the speaker, methods, pros and cons of the application proposed.

Table 1: A Comparison of Classification algorithms in PCOS detection system

\begin{tabular}{|c|c|c|c|}
\hline Citation & Method & Advantages & Disadvantages \\
\hline [31] & $\begin{array}{l}\text { For segmentation of follicles, } \\
\text { adaptive k-means clustering is } \\
\text { applied. Statistical GLCM is } \\
\text { employed to extract features and } \\
\text { these features are used to train the } \\
\text { ANN. }\end{array}$ & $\begin{array}{l}\text { The proposed system achieved } \\
\text { better follicle detection results } \\
\text { with high accuracy rate. It is not } \\
\text { affected by noise or errors in } \\
\text { the training data. }\end{array}$ & $\begin{array}{l}\text { The parameters of } \\
\text { the ANN can be } \\
\text { optimized using } \\
\text { different } \\
\text { algorithms }\end{array}$ \\
\hline [32] & $\begin{array}{l}\text { The use of active contour with } \\
\text { modified Otsu threshold value was } \\
\text { implemented to automatically detect } \\
\text { follicles in ultrasound images. }\end{array}$ & High accuracy & $\begin{array}{lr}\text { Large } & \text { database } \\
\text { cannot } & \text { be } \\
\text { applicable } & \end{array}$ \\
\hline [33] & $\begin{array}{l}\text { PCOS recognition using an SVM } \\
\text { kernel, with a probabilistic model to } \\
\text { learn the distribution of the derived } \\
\text { feature vector. }\end{array}$ & $\begin{array}{l}\text { Changes in endometrial } \\
\text { thickness and blood flow can be } \\
\text { identified through medical } \\
\text { imaging, allowing early } \\
\text { prediction and treatment of } \\
\text { disorders. }\end{array}$ & $\begin{array}{l}\text { Accuracy can be } \\
\text { improved }\end{array}$ \\
\hline [34] & $\begin{array}{l}\text { Different image processing } \\
\text { techniques are applied to ultrasound } \\
\text { images of ovary. Canny edge } \\
\text { detection method is used to identify } \\
\text { the follicle edges. }\end{array}$ & $\begin{array}{l}\text { Proposed techniques are more } \\
\text { appropriate for extraction and } \\
\text { detection of follicles from the } \\
\text { ovary images. }\end{array}$ & $\begin{array}{l}\text { Different kind of } \\
\text { image dataset } \\
\text { cannot be used }\end{array}$ \\
\hline [35] & $\begin{array}{l}\text { Gabor Wavelet method for feature } \\
\text { extraction and competitive neural } \\
\text { network for classification }\end{array}$ & Accuracy is high & $\begin{array}{l}\text { Large number of } \\
\text { dataset cannot be } \\
\text { used }\end{array}$ \\
\hline
\end{tabular}




\begin{tabular}{|c|c|c|c|}
\hline$[36]$ & $\begin{array}{l}\text { Gabor Wavelet method was used to } \\
\text { extract features and Elman Neural } \\
\text { Network acted as the classifier. }\end{array}$ & $\begin{array}{l}\text { The classifier has better } \\
\text { performance }\end{array}$ & $\begin{array}{l}\text { The large number } \\
\text { of data affect the } \\
\text { time process }\end{array}$ \\
\hline$[37]$ & $\begin{array}{l}\text { Before extracting features, the } \\
\text { ultrasound images were despeckled } \\
\text { with a Lee filter. }\end{array}$ & $\begin{array}{l}\text { It is suitable for routine } \\
\text { segmentation }\end{array}$ & $\begin{array}{lr}\text { Follicle detection } \\
\text { rate can be } \\
\text { increased } & \text { using } \\
\text { different } & \\
\text { algorithms } & \\
\end{array}$ \\
\hline [38] & $\begin{array}{lcr}\text { Proposed Levenberg } & \text { Marquardt } \\
\text { optimization } & \text { and } & \text { Conjugate } \\
\text { Gradient } & \text { Fletcher } & \text { Reeves } \\
\text { algorithms } & \text { to } & \text { improve } \\
\text { backpropagation algorithm. }\end{array}$ & It has high accuracy & $\begin{array}{l}\text { Conjugate gradient } \\
\text { parameter is not } \\
\text { considered in this } \\
\text { work }\end{array}$ \\
\hline [39] & $\begin{array}{l}\text { Particle Swarm Optimization (PSO) } \\
\text { and a modified nonparametric } \\
\text { fitness function for segmentation of } \\
\text { follicles. }\end{array}$ & $\begin{array}{l}\text { Contrast enhancement can } \\
\text { improve the extracted follicular } \\
\text { size to be closer toward to the } \\
\text { actual follicular size. }\end{array}$ & $\begin{array}{l}\text { The follicles cannot } \\
\text { be identified } \\
\text { automatically }\end{array}$ \\
\hline$[40]$ & $\begin{array}{l}\text { To identify smaller follicles, active } \\
\text { contour without edge method was } \\
\text { proposed. Spilt-Bregman } \\
\text { optimization reduces the time } \\
\text { required for the process. }\end{array}$ & The performance is high & $\begin{array}{ll}\text { The accuracy } & \text { can } \\
\text { be increased by } \\
\text { using } & \text { an } \\
\text { optimization } & \\
\text { algorithm } & \end{array}$ \\
\hline [41] & $\begin{array}{l}\text { Bayesian classifier was used to as } \\
\text { classification algorithm. Only four } \\
\text { classifiers were used. }\end{array}$ & $\begin{array}{l}\text { The accuracy was increased to } \\
93.93 \% \text { compared with logical } \\
\text { regression classifiers. }\end{array}$ & $\begin{array}{l}\text { The accuracy can } \\
\text { be enhanced with } \\
\text { other classifiers. }\end{array}$ \\
\hline [42] & $\begin{array}{l}\text { The authors used PCA feature } \\
\text { transformation and statistical } \\
\text { significance. For classification, } \\
\text { naïve Bayes classifier, logistic } \\
\text { regression, KNN, Classification and } \\
\text { Regression Trees (CART), random } \\
\text { forest classifier, and SVM were } \\
\text { used. }\end{array}$ & $\begin{array}{l}\mathrm{RF} \text { algorithm is found superior } \\
\text { in performance }\end{array}$ & $\begin{array}{l}\text { Biochemical and } \\
\text { laboratory results } \\
\text { only were used. }\end{array}$ \\
\hline [43] & $\begin{array}{l}\text { Clinical image parameters were } \\
\text { analysed using Demon and free form } \\
\text { Deformation method }\end{array}$ & $\begin{array}{l}\text { Demon algorithm for complex } \\
\text { motion images }\end{array}$ & $\begin{array}{l}\text { The performance } \\
\text { can be improved } \\
\text { with incorporating } \\
\text { machine learning } \\
\text { algorithms }\end{array}$ \\
\hline$[44]$ & $\begin{array}{l}\text { SVM, CART, logistic regression, } \\
\text { random forest and naive Bayes } \\
\text { classifier were used for study. }\end{array}$ & $\begin{array}{l}\text { Found that Random Forest } \\
\text { algorithm have } 96 \% \text { accuracy }\end{array}$ & $\begin{array}{l}\text { Only text data was } \\
\text { analysed }\end{array}$ \\
\hline [45] & Segmentation is used prior $\mathrm{CNN}$ & $\begin{array}{l}\text { To delete or eliminate } \\
\text { redundant data. }\end{array}$ & $\begin{array}{l}\text { Only image is } \\
\text { considered }\end{array}$ \\
\hline
\end{tabular}

\section{RESEARCH GAP :}

In the past few years, researchers focused on meta-heuristic, heuristic, and optimization approaches are used for PCOS detection. PCOS has become the most common hormonal disorder among females. In recent research, different feature selection as well as different classification techniques were used for achieves better results. PCOS in women may be correlated with menstrual irregularities, hormonal imbalance, hirsutism, obesity, acne and other variables. The primary method of detecting ovarian follicles is analysis of ultrasound images of the ovaries. Tasks such as detecting of follicles, foetal growth detection, foetal development monitoring and tumour diagnosis are all non-trivial that requires the application of object detection in ultrasound images. Presently, doctors diagnose PCOS by analysing ultrasound images of the ovaries and manually counting the number of follicular cysts to see if the syndrome is present. 


\subsection{Research Gap 1:}

Manual counting can cause challenges with consistency, reproducibility, and efficiency. Also, the existing techniques cannot identify the PCOS disease from adolescence. Variability, reproducibility, and efficiency issues may arise as a result of manual counting.

\subsection{Research Gap 2:}

The quality of the ultrasound image is an essential part for the classification. An algorithm developed for cyst classification may not be applicable for all types of cysts, as there are great variations in the features of cysts. Researchers are now advocating the nexus of Artificial Intelligence (AI) with medical imaging, to enable automatic classification of ultrasound images. AI can learn features from very large amount of medical data acquired from clinical practice to diagnose diseases accurately. AI can also filter and clean the data and to detect the disease with high accuracy and precision. It must be noted that the boundary of the ovaries may not be clearly visible in some cases and if follicles appear as local minima, it will be difficult to identify them. Features such as the characteristics of the ovary region and the connectivity between follicles are not considered by the classification models.

\subsection{Research Gap 3:}

In the recent studies, the ovaries' boundary is not clearly defined and follicles can appear as local minima, these approaches are inadequate for follicle detection. The properties of the ovary region, as well as the connectivity or proximity between follicles, were not taken into account by these classification-based techniques. It is necessary to consider the attributes of follicles, the ovary's boundary information, and the different area information in the ovary ultrasound image to produce improved follicle identification results. Also, the existing techniques cannot classify the all kinds of cysts.

To address these issues, this study presents a system for the early identification and prediction of PCOS based on a set of ideal and minimal, but promising, clinical and metabolic indicators that can serve as early markers for the condition.

\section{PROPOSED ARCHITECTURE FOR PCOS DETECTION :}

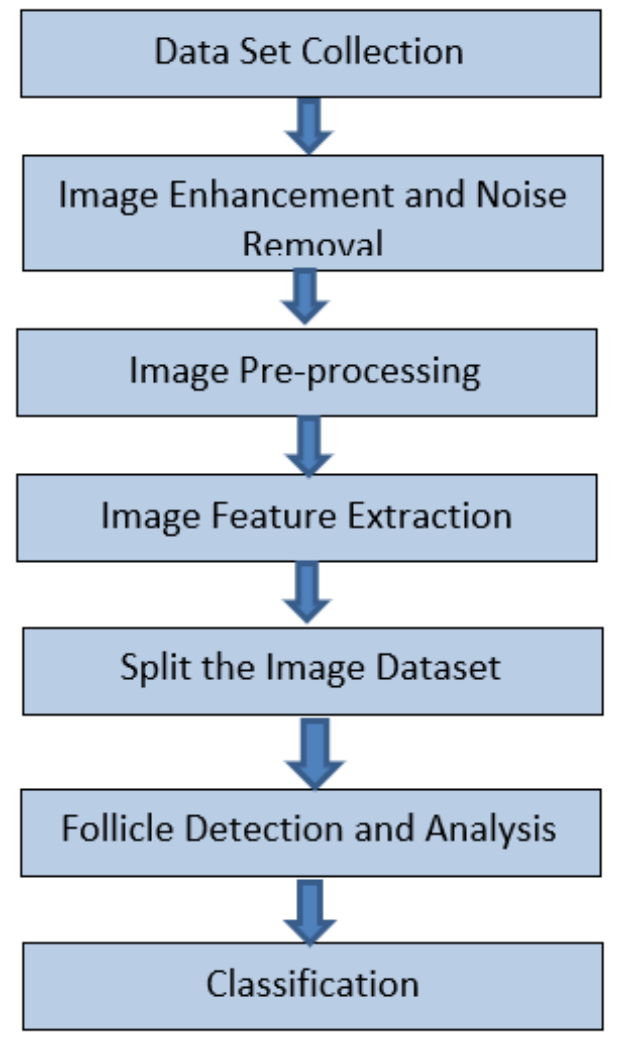

Fig. 6: Hierarchical Structure of PCOS Detection 
Firstly, the dataset is collected and pre-processed. Later, the quality of the image collected has to be enhanced. Later on, segmentation is done using clustering algorithm with fuzzy filter. The classification can be achieved by using attention based CNN-RNN deep model. A novel machine learning method is proposed for the prediction of PCOS. The text data collected from the survey is proposed to be used for prediction.

\section{RESEARCH AGENDA :}

(1) Which are the best algorithms to classify PCOS?

(2) How the quality of the image can be enhanced?

(3) What system can be developed to increase the privacy of the patient?

(4) What are the challenges in the implementation of different algorithms in development of automatic detection systems?

(5) What new technology can be proposed for implementation perspective?

\section{ANALYSIS OF RESEARCH AGENDA :}

The different approaches and methodology can be used to analyse the data collected. The most of the existing algorithms are less concise. This research aims to develop a hybrid algorithm to yield best effective classification results. The quality of the raw image collected has to be improved using the noise removal algorithms, as it directly affects the preciseness of the study. The privacy of the patients was a major challenge during the study. It can be ensured by keeping their personal details hidden and only making use of only the details which are required for the prediction of PCOS. The collection of data for the different types of PCOS is a challenging. The early stage prediction of the syndrome with high accuracy is a big challenge and has to be solved in the later stage. The early stage prediction of the syndrome will be a great blessing as they can reduce the change of getting affected by adopting changes in their daily life routine. The development of automatic detection of the syndrome will be very helpful for the doctors as manual counting is not required and the result is achieved with high accuracy.

\section{FINAL RESEARCH PROPOSAL IN CHOSEN TOPIC :}

A sophisticated novel approach for PCOS prediction and detection using machine learning techniques.

\section{ABCD ANALYSIS OF RESEARCH PROPOSAL :}

ABCD methodology [51-52] is used to examine the features of the system and the potencies of the strategic ideas which are supposed to be implemented in the society. Based on four constructs - Advantages, Benefits, Constraints, and Disadvantages - the system and its features are analysed and the major issues are identified [53].

\subsection{Advantages:}

(1) It provides a genuine method for the medical practitioners.

(2) Prediction system helps the female category to adopt appropriate change in their lifestyle for good health.

(3) Expels the hurdles faced in traditional diagnosis techniques.

\subsection{Benefits:}

(1) Beneficial for the all the gynaecologists and endocrinologists.

(2) Prediction system helps the ladies in finding out the lifestyle they have to adopt.

\subsection{Constraints:}

(1) The availability of data set of different category of patients is difficult.

(2) The complete transformation from traditional approach to automated system.

(3) Selection of appropriate algorithm for image analysis.

(4) Prediction result may be affected by the survey data.

\subsection{Disadvantages:}

(1) The exactness of the approach relies upon the accessible information.

(2) Any variation in the result of prediction can lead to misinterpretation.

(3) The result of the detection system if false can lead to wrong treatment procedures. 


\section{CONCLUSION :}

Polycystic Ovary Syndrome (PCOS) has become the common form of endocrine disorder in women of reproductive age and can result in anovulation leading to infertility. PCOS diagnosis is based on both clinical and metabolic parameters, which serve as biomarkers for the syndrome. Classification techniques are considered in this study as it enables us to predict if the patient has Polycystic Ovarian Syndrome or not based on the syndromes provided by the doctor or medical centre. A sophisticated novel approach using machine learning techniques is proposed to be implemented for better results. Currently there are so many uses of techniques, methods and tools to detect PCOS and further research is going on to get an efficient CAD system. In this review paper, we have done a systematic study on the classification techniques and medical image processing methodologies. Based on the study we realized that the algorithms which were implemented in many of the cited research works was merely done either on the image or on the text data. As a consequence of this, only classification was possible. The performance may vary according to different algorithms and input dataset. Medical image processing phases, techniques, features to be extracted; classifiers used are also discussed in this paper. As a result of the study, we can claim that the sophisticated machine learning technologies are capable to identify anomalies through proper training.

\section{REFERENCES :}

[1] Nazarudin, A. A., Zulkarnain, N., Hussain, A., Mokri, S. S., \& Nordin, I. N. (2020). Review on automated follicle identification for polycystic ovarian syndrome. Bulletin of Electrical Engineering and Informatics, 9(2), 588-593.

\section{Google Scholar $\chi^{\nearrow} \quad$ CrossRef/DOI $\chi^{\nearrow}$}

[2] Palm, C. V., Glintborg, D., Kyhl, H. B., McIntyre, H. D., Jensen, R. C., Jensen, T. K., Jensen, D. M., \& Andersen, M. (2018). Polycystic ovary syndrome and hyperglycaemia in pregnancy. A narrative review and results from a prospective Danish cohort study. Diabetes Research and Clinical Practice, 145, 167177.

\section{Google Scholar $x^{\nearrow} \quad \underline{\text { CrossRef/DOIX }}$}

[3] Escobar-Morreale, H. F. (2018). Polycystic ovary syndrome: Definition, aetiology, diagnosis and treatment. Nature Reviews Endocrinology, 14(5), 270-284.

Google Scholar $x^{7} \quad$ CrossRef/DOI $\chi^{\top}$

[4] Otaghi, M., Azami, M., Khorshidi, A., Borji, M., \& Tardeh, Z. (2019). The association between metabolic syndrome and polycystic ovary syndrome: A systematic review and meta-analysis. Diabetes \& Metabolic Syndrome: Clinical Research \& Reviews, 13(2), 1481-1489.

\section{Google Scholar $X^{\nearrow} \quad$ CrossRef/DOIX}

[5] Tassone, E., Teede, H., Dokras, A., Garad, R., \& Gibson-Helm, M. (2018). The needs of women and healthcare providers regarding polycystic ovary syndrome information, resources, and education: A systematic search and narrative review. Seminars in Reproductive Medicine, 36(01), 035-041.

Google Scholar $\chi^{7} \quad \underline{\text { CrossRef/DOI } \chi^{7}}$

[6] Soni, P., \& Vashisht, S. (2019). Image segmentation for detecting polycystic ovarian disease using deep neural networks. International Journal of Computer Sciences and Engineering, 7(3), 534-537. Google Scholar $\chi^{\nearrow} \quad$ CrossRef/DOI $\chi^{\nearrow}$

[7] Patel, S. (2018). Polycystic ovary syndrome (PCOS), an inflammatory, systemic, lifestyle endocrinopathy. The Journal of Steroid Biochemistry and Molecular Biology, 182(1), 27-36. Google Scholar $X^{7} \quad$ CrossRef/DOIX

[8] Zhang, Q., Bao, Z.-kun, Deng, M.-xiang, Xu, Q., Ding, D.-dan, Pan, M.-man, Xi, X., Wang, F.-fang, Zou, Y., \& Qu, F. (2020). Fetal growth, fetal development, and placental features in women with polycystic ovary syndrome: Analysis based on fetal and placental magnetic resonance imaging. Journal of Zhejiang University-SCIENCE B, 21(12), 977-989.

Google Scholar $\chi^{\nearrow} \quad$ CrossRef/DOI $\chi^{\nearrow}$

[9] Zeinalabdeen, M. Z., \& Gar-Elnabi, M. E. (2020). Characterization of infertility using ultrasonography. Scholars Journal of Applied Medical Sciences, 8(10), 2326-2330. Google Scholar $x^{7} \quad$ CrossRef/DOIX 
[10] Kumar, K. S., Nirmala, V., Venkatalakshmi, K., \& Karthikeyan, K. (2018). Analysis of optimization algorithms on follicles segmentation to support polycystic ovarian syndrome detection. Journal of Computational and Theoretical Nanoscience, 15(1), 380-391.

Google Scholar $x^{7} \quad$ CrossRef/DOI $x^{7}$

[11] Sumathi, M., Chitra, P., Sakthi Prabha, R., \& Srilatha, K. (2021). Study and detection of PCOS related diseases using CNN. IOP Conference Series: Materials Science and Engineering, 1070(1), 012-062. Google Scholar $X^{\top}$

CrossRef/DOI $x^{\nearrow}$

[12] Jarrett, B. Y., Vanden Brink, H., Oldfield, A. L., \& Lujan, M. E. (2020). Ultrasound characterization of disordered antral follicle development in women with polycystic ovary syndrome. The Journal of Clinical Endocrinology \& Metabolism, 105(11), e3847-e3861.

Google Scholar $x^{7} \quad$ CrossRef/DOI $\chi^{\nearrow}$

[13] YILMAZ, P. G., \& ÖZMEN, G. (2020). Follicle detection for polycystic ovary syndrome by using image processing methods. International Journal of Applied Mathematics Electronics and Computers, 8(4), 203-208.

Google Scholar $X^{7}$

CrossRef/DOIX

[14] Srivastava, S., Kumar, P., Chaudhry, V., \& Singh, A. (2020). Detection of ovarian cyst in ultrasound images using fine-tuned VGG-16 Deep Learning Network. SN Computer Science, 1(2), 1-8. Google Scholar $\chi^{\nearrow} \quad$ CrossRef/DOI $\chi^{\nearrow}$

[15] Satish, C. N., Chew, X., \& Khaw, K. W. (2020). Polycystic Ovarian Syndrome (PCOS) classification and feature selection by machine learning techniques, Applied Mathematics and Computational Intelligence, 9(1), 65-74.

Google Scholar $x^{7}$

[16] Deepika, V. (2019). Applications of Artificial Intelligence Techniques in Polycystic ovarian syndrome Diagnosis. Journal of Advanced Research in Technology and Management Sciences, 1(3), 59-63. Google Scholar ${ }^{\top}$

[17] Bharati, S., Podder, P., \& Hossain Mondal, M. R. (2020). Diagnosis of polycystic ovary syndrome using machine learning algorithms. 2020 IEEE Region 10 Symposium (TENSYMP). (pp. 1486-1489). IEEE. Google Scholar Х $\quad$ CrossRef/DOIX

[18] Denny, A., Raj, A., Ashok, A., Ram, C. M., \& George, R. (2019, October). I-HOPE: detection and prediction system for polycystic ovary syndrome (PCOS) using machine learning techniques. In TENCON 2019-2019 IEEE Region 10 Conference (TENCON) (pp. 673-678). IEEE.

Google Scholar $X^{7} \quad$ CrossRef/DOIX

[19] Ramamoorthy, S., \& Sivasubramaniam, R. (2019, January). Monitoring the growth of Polycystic Ovary Syndrome using Mono-modal Image Registration Technique: Application of Medical Big Data in Healthcare. In Proceedings of the ACM India Joint International Conference on Data Science and Management of Data, 180-187.

Google Scholar $\chi^{\top}$

CrossRef/DOI $x^{7}$

[20] Fu, Y., Lei, Y., Wang, T., Curran, W. J., Liu, T., \& Yang, X. (2020). Deep learning in medical image registration: a review. Physics in Medicine \& Biology, 65(20), 20 TR01.

Google Scholar $\chi^{\nearrow} \quad$ CrossRef/DOI $\chi^{\nearrow}$

[21] Bozdag, G., Mumusoglu, S., Zengin, D., Karabulut, E., \& Yildiz, B. O. (2016). The prevalence and phenotypic features of polycystic ovary syndrome: A systematic review and meta-analysis. Human Reproduction, 31(12), 2841-2855.

Google Scholar $\chi^{7} \quad \underline{\text { CrossRef/DOIX }}$

[22] Cesta, C. E., Öberg, A. S., Ibrahimson, A., Yusuf, I., Larsson, H., Almqvist, C., D'Onofrio, B. M., Bulik, C. M., Fernández de la Cruz, L., Mataix-Cols, D., Landén, M., \& Rosenqvist, M. A. (2019). Maternal polycystic ovary syndrome and risk of neuropsychiatric disorders in offspring: Prenatal androgen exposure or genetic confounding? Psychological Medicine, 50(4), 616-624.

Google Scholar $X^{\nearrow} \quad \underline{\text { CrossRef/DOIX }}$ 
[23] Huijgen, N. A., de Ridder, M. A. J., Verhamme, K. M., Dohle, G. R., Vanrolleghem, A. M., Sturkenboom, M. C. J. M., Laven, J. S. E., \& Steegers-Theunissen, R. P. M. (2016). Are proton-pump inhibitors harmful for the semen quality of men in couples who are planning pregnancy? Fertility and Sterility, 106(7),1666-1672.

Google Scholar 7 CrossRef/DOIX

[24] Doherty, D. A., Newnham, J. P., Bower, C., \& Hart, R. (2015). Implications of polycystic ovary syndrome for pregnancy and for the health of offspring. Obstetrics \& Gynecology, 125(6), 1397-1406. Google Scholar $x^{7}$

CrossRef/DOI $x^{\nearrow}$

[25] Goodarzi, M., Chazenbalk, G., Abbott, D., \& Dumesic, D. (2014). Intrauterine environment and polycystic ovary syndrome. Seminars in Reproductive Medicine, 32(03), 159-165.

Google Scholar $\chi^{\nearrow} \quad \underline{\text { CrossRef/DOIX }}$

[26] Hjorth-Hansen, A., Salvesen, Ø., Engen Hanem, L. G., Eggebø, T., Salvesen, K. Å., Vanky, E., \& Ødegård, R. (2017). Fetal growth and birth anthropometrics in metformin-exposed offspring born to mothers with PCOS. The Journal of Clinical Endocrinology \& Metabolism, 103(2), 740-747.

Google Scholar $\chi^{\nearrow} \quad$ CrossRef/DOIX

[27] Kelley, A. S., Smith, Y. R., \& Padmanabhan, V. (2019). A narrative review of placental contribution to adverse pregnancy outcomes in women with polycystic ovary syndrome. The Journal of Clinical Endocrinology \& Metabolism, 104(11), 5299-5315.

Google Scholar $\chi^{\nearrow} \quad$ CrossRef/DOI $\chi^{\nearrow}$

[28] Li, R., Zhang, Q., Yang, D., Li, S., Lu, S., Wu, X., Wei, Z., Song, X., Wang, X., Fu, S., Lin, J., Zhu, Y., Jiang, Y., Feng, H. L., \& Qiao, J. (2013). Prevalence of polycystic ovary syndrome in women in China: A large community-based study. Human Reproduction, 28(9), 2562-2569.

Google Scholar ${ }^{\nearrow} \quad$ CrossRef/DOI $\chi^{\nearrow}$

[29] Maliqueo, M., Sundstrom Poromaa, I., Vanky, E., Fornes, R., Benrick, A., Akerud, H., Stridsklev, S., Labrie, F., Jansson, T., \& Stener-Victorin, E. (2015). Placental STAT3 signaling is activated in women with polycystic ovary syndrome. Human Reproduction, 30(3), 692-700.

Google Scholar ${ }^{7}$

CrossRef/DOIX

[30] McDonnell, R., \& Hart, R. J. (2017). Pregnancy-related outcomes for women with polycystic ovary syndrome. Women's Health, 13(3), 89-97.

Google Scholar 7 CrossRef/DOIX

[31] Nilofer, N. S. (2021). Follicles Classification To Detect Polycystic Ovary Syndrome Using Glcm And Novel Hybrid Machine Learning. Turkish Journal of Computer and Mathematics Education (TURCOMAT), 12(7), 1062-1073.

Google Scholar $X^{7} \quad \underline{\text { CrossRef/DOIX }}$

[32] Gopalakrishnan, C., \& Iyapparaja, M. (2019). Active contour with modified Otsu method for automatic detection of polycystic ovary syndrome from ultrasound image of ovary. Multimedia Tools and Applications, 79(23), 17169-17192.

Google Scholar 7 CrossRef/DOI $\chi^{7}$

[33] He, D., Liu, L., Miao, S., Tong, X., \& Sheng, M. (2019). Probabilistic guided polycystic ovary syndrome recognition using learned quality kernel. Journal of Visual Communication and Image Representation, 63(1), 102587.

Google Scholar $7 \quad$ CrossRef/DOIX

[34] Gopalakrishnan, C., \& Iyapparaja, M. (2019). Detection of polycystic ovary syndrome from ultrasound images using SIFT descriptors. Bonfring International Journal of Software Engineering and Soft Computing, 9(2), 26-30.

Google Scholar $x^{7}$

\section{CrossRef/DOIX}

[35] Dewi, R. M., Adiwijaya, Wisesty, U. N., \& Jondri. (2018). Classification of polycystic ovary based on ultrasound images using competitive neural network. Journal of Physics: Conference Series, 971(1), 012-025. 


\section{Google Scholar $X^{\nearrow} \quad \underline{\text { CrossRef/DOIX }}$}

[36] Thufailah, I. F., Adiwijaya, Wisesty, U. N., \& Jondri. (2018). An implementation of Elman Neural Network for polycystic ovary classification based on ultrasound images. Journal of Physics: Conference Series, 971(1), 012-016.

Google Scholar $\chi^{7}$

\section{CrossRef/DOIX}

[37] Isah, O. R., Usman, A. D., \& Tekanyi, A. M. S. (2017). A Hybrid Model of PSO Algorithm and Artificial Neural Network for Automatic Follicle Classification. International Journal Bioautomation, 21(1), 4358 .

Google Scholar $x^{7}$

[38] Wisesty, U. N., Nasri, J., \& Adiwijaya. (2016). Modified backpropagation algorithm for polycystic ovary syndrome detection based on ultrasound images. Advances in Intelligent Systems and Computing, 2(2), $141-151$.

Google Scholar 7 CrossRef/DOIX

[39] Setiawati, E., Adiwijaya, \& Tjokorda, A. B. (2015). Particle swarm optimization on follicles segmentation to support PCOS detection. 2015 3rd International Conference on Information and Communication Technology (ICoICT), 3(2), 369-374.

Google Scholar $x^{7} \quad \underline{\text { CrossRef/DOIX }}$

[40] Kumar, H. P., \& Srinivasan, S. (2015). Fast automatic segmentation of polycystic ovary in ultrasound images using improved Chan-vase with split-bregman optimization. Journal of Medical Imaging and Health Informatics, 5(1), 57-62.

Google Scholar 7 CrossRef/DOIX

[41] Mehrotra, P., Chatterjee, J., Chakraborty, C., Ghoshdastidar, B., \& Ghoshdastidar, S. (2011, December). Automated screening of polycystic ovary syndrome using machine learning techniques. In 2011 Annual IEEE India Conference (pp. 1-5). IEEE.

Google Scholar $\chi^{\nearrow} \quad \underline{\text { CrossRef/DOIX }}$

[42] Denny, A., Raj, A., Ashok, A., Ram, C. M., \& George, R. (2019, October). I-HOPE: detection and prediction system for polycystic ovary syndrome (PCOS) using machine learning techniques. In TENCON 2019-2019 IEEE Region 10 Conference (TENCON) (pp. 673-678). IEEE.

Google Scholar $X^{7} \quad$ CrossRef/DOI $\chi^{\nearrow}$

[43] Schnabel, J. A., Heinrich, M. P., Papież, B. W., \& Brady, S. J. (2016). Advances and challenges in deformable image registration: From image fusion to complex motion modelling. Medical Image Analysis, 33(3), 145-148.

Google Scholar $x^{7} \quad \underline{\text { CrossRef/DOI }} \chi^{7}$

[44] Mubasher Hassan, M., \& Mirza, T. (2020). Comparative analysis of machine learning algorithms in diagnosis of polycystic ovarian syndrome. International Journal of Computer Applications, 175(17), $42-53$.

Google Scholar $x^{7} \quad$ CrossRef/DOIX

[45] Soni, P., \& Vashisht, S. (2019). Image segmentation for detecting polycystic ovarian disease using deep neural networks. International Journal of Computer Sciences and Engineering, 7(3), 534-537.

Google Scholar ${ }^{\nearrow} \quad$ CrossRef/DOI $\chi^{\nearrow}$

[46] Huang, Q., Zhang, F., \& Li, X. (2018). Machine learning in ultrasound computer-aided diagnostic systems: A survey. BioMed Research International, 1(1), 1-10.

Google Scholar $X^{\top} \quad \underline{\text { CrossRef/DOIX' }}$

[47] Gomez, W., Pereira, W. C., \& Infantosi, A. F. (2012). Analysis of co-occurrence texture statistics as a function of gray-level quantization for classifying breast ultrasound. IEEE Transactions on Medical Imaging, 31(10), 1889-1899.

Google Scholar ${ }^{\top}$

\section{CrossRef/DOIX}

[48] Zhang, Q., Li, C., Han, H., Dai, W., Shi, J., Wang, Y., \& Wang, W. (2015). Spatio-temporal quantification of carotid plaque neovascularization on contrast enhanced ultrasound: Correlation with 
visual grading and histopathology. European Journal of Vascular and Endovascular Surgery, 50(3), 289-296.

Google Scholar $\chi^{7} \quad$ CrossRef/DOI $\chi^{\nearrow}$

[49] Xiao, L., Shao, Z., \& Liu, G. (2006, June). K-means algorithm based on particle swarm optimization algorithm for anomaly intrusion detection. In 2006 6th World Congress on Intelligent Control and Automation, 2(1), 5854-5858. IEEE.

Google Scholar 7 CrossRef/DOIX

[50] Sahoo, T. K., \& Banka, H. (2017). New hybrid PCA-based facial age estimation using inter-age group variation-based hierarchical classifier. Arabian Journal for Science and Engineering, 42(8), 3337-3355. Google Scholar ${ }^{\nearrow} \quad$ CrossRef/DOI ${ }^{\nearrow}$

[51] Aithal, P. S., \& Aithal, S. (2018). Factor \& Elemental Analysis of Nanotechnology as Green Technology using ABCD Framework. International Journal of Management, Technology, and Social Sciences (IJMTS), 3(2), 57-72.

Google Scholar 7

[52] Aithal, P. S., Shailashree, V., \& Kumar, P. M. (2015). A new ABCD technique to analyze business models \& concepts. International Journal of Management, IT and Engineering, 5(4), 409-423. Google Scholar $\nearrow^{\top}$

[53] Aithal, P. S. (2017). ABCD Analysis as Research Methodology in Company Case Studies. International Journal of Management, Technology, and Social Sciences (IJMTS), 2(2), 40-54.

Google Scholar X 